\title{
Phenomenological aspects of kaon photoproduction on the nucleon
}

\author{
T. Mart ${ }^{\mathrm{a} *}$, S. Sumowidagdo ${ }^{\mathrm{a} *}$, C. Bennhold ${ }^{\mathrm{b} \dagger}$, and H. Haberzettl ${ }^{\mathrm{b} \dagger}$ \\ a Jurusan Fisika, FMIPA, Universitas Indonesia, Depok 16424, Indonesia \\ ${ }^{\mathrm{b}}$ Center for Nuclear Studies, Department of Physics, The George Washington University, \\ Washington, D.C. 20052, USA \\ Using an isobar model which can reproduce the existing experimental data of kaon \\ photoproduction on the nucleon we investigate some related phenomenological aspects, \\ i.e. the hadronic form factors, contributions of kaon-hyperon final states to the anomalous \\ magnetic moment of the nucleon, and missing nucleon resonances. By reggeizing the \\ appropriate propagators we extend the model to the higher photon energy regime.
}

\section{INTRODUCTION}

A wealth of new high-statistics data on elementary kaon photo- and electroproduction has recently become available in three isospin channels. Along with some new progress in the theoretical side this has made the field of kaon electromagnetic production to be of considerable interest. New models, span from chiral perturbation theory to the relatively simple isobar approach, have been proposed in the recent years as the SAPHIR collaboration made their precise data publicly available. Because the error-bars are sufficiently small, an interesting structure can be resolved in $K^{+} \Lambda$ total cross section. This leads to a critical question, as to whether the structure comes from less known resonances or other reaction channels start to open at the corresponding energy.

In this paper we discuss some phenomenological aspects, which can be investigated by means of the isobar model. The model has been constructed by including three states that have been found to have significant decay widths into $K \Lambda$ and $K \Sigma$ channels, the $S_{11}(1650), P_{11}(1710)$, and $P_{13}(1720)$ resonances, to fit all elementary data by adjusting some free parameters, which are known as coupling constants.

\section{THE MODEL AND SOME PHENOMENOLOGICAL ASPECTS}

\subsection{Hadronic Form Factors}

Previous analyses of kaon photoproduction have never included a form factor at the hadronic vertex. However, since most of the present isobaric models diverge at higher energies, the need for such hadronic form factors has been known for a long time. Furthermore, it has been demonstrated that models which give a good description of the $\left(\gamma, K^{+}\right)$data can give unrealistically large predictions for the $\left(\gamma, K^{0}\right)$ channels [1]. It

\footnotetext{
*Supported in part by the University Research for Graduate Education (URGE) grant.

${ }^{\dagger}$ Supported in part by US DOE with grant no. DE-FG02-95ER-40907
} 
is well known that incorporating a hadronic form factor helps alleviate this divergence and, simultaneously, leads to a problem with gauge invariance, since not every diagram in the Born terms retains gauge invariance by itself." The question of gauge invariance is actually one of the central issues in dynamical descriptions of how photons interact with hadronic systems. While there is usually no problem at the tree-level with bare, pointlike particles, the problem becomes very complicated once the electromagnetic interaction is consistently incorporated within the full complexity of a strongly-interacting hadronic system.

In the previous work [2] we have studied the influence of hadronic form factors on kaon production, by multiplying the whole amplitude with an overall, monopole, form factor $F(t)$, i.e.

$M_{\mathrm{fi}}=\left[M_{\mathrm{B}}(s, t, u)+M_{\mathrm{R}}(s, t, u)\right] \cdot F(t)$,

where the subscripts $\mathrm{B}$ and $\mathrm{R}$ refer to the Born and resonance terms, to simulate the average effect of the fact that nucleons are not point-like. In spite of the success to suppress the divergence and to avoid the problem of gauge invariance, this ad hoc fashion does not have any microscopic foundation.

In order to restore gauge invariance properly, one needs to construct additional current contributions beyond the usual Feynman diagrams to cancel the gauge-violating terms. One of the most widely used methods is due to Ohta [3]. For kaon photoproduction off the nucleon, Ohta's prescription amounts to dropping all strong-interaction form factors for all gauge-violating electric current contributions in Born terms. Symbolically, this may be written as

$M_{\mathrm{B}}(s, t, u)=M_{\mathrm{B}}^{\text {mag. }}[s, t, u, F(s), F(t), F(u)]+M_{\mathrm{B}}^{\text {elec. }}(s, t, u)$.

The recipe, however, does not completely solve the problem of divergence, since the electric terms do not have suppression and, therefore, could violently increase as a function of the coupling constants. As shown in Ref. [4], even at the coupling constants values accepted by the $\mathrm{SU}(3)$ symmetry, Ohta's recipe already yields very large $\chi^{2}$.

On the other hand, Haberzettl [5] has put forward a comprehensive treatment of gauge invariance in meson photoproduction. This includes a prescription for restoring gauge invariance in situations when one cannot handle the full complexity of the problem and therefore must resort to some approximations. In our language, this method can be translated as

$M_{\mathrm{B}}(s, t, u)=M_{\mathrm{B}}^{\text {mag. }}[s, t, u, F(s), F(t), F(u)]+M_{\mathrm{B}}^{\text {elec. }}(s, t, u) \cdot \hat{F}(s, t, u)$,

with $\hat{F}(s, t, u)=a_{1} F(s)+a_{2} F(t)+a_{3} F(u)$ and $a_{1}+a_{2}+a_{3}=1$. Clearly, Haberzettl's method removes the Ohta's problem by an additional form factor in the electric terms.

By fitting to the kaon photoproduction data we found that the method proposed by Haberzettl to be superior rather than the Ohta's, since the former can provide a reasonable description of the data using values for the leading couplings constants close to the $\mathrm{SU}(3)$ prediction. Such couplings cannot be accommodated in Ohta's method due to the absence of a hadronic form factor in the electric current contribution.

\footnotetext{
${ }^{3}$ Since the resonance terms are individually gauge invariant, the discussion will be limited to the Born terms.
} 
Table 1

Numerical values for the contribution of kaon-hyperon final states to the square of anomalous magnetic moments of proton and neutron. Column (1) is obtained from Eq. (5), while column (2) is evaluated by using Eq. (6). Experimentally, $\kappa_{p}^{2}=3.214$ and $\kappa_{n}^{2}=3.660$.

\begin{tabular}{|c|c|c|c|c|c|}
\hline \multirow[b]{2}{*}{ Channel } & \multicolumn{2}{|c|}{$\kappa_{p}^{2}(K)$} & \multirow[b]{2}{*}{ Channel } & \multicolumn{2}{|c|}{$\kappa_{n}^{2}(K)$} \\
\hline & (1) & $(2)$ & & (1) & $(2)$ \\
\hline$\gamma p \rightarrow K^{+} \Lambda$ & -0.026 & 0.044 & $\gamma n \rightarrow K^{0} \Lambda$ & 0.075 & 0.110 \\
\hline$\gamma p \rightarrow K^{+} \Sigma^{0}$ & -0.024 & 0.030 & $\gamma n \rightarrow K^{+} \Sigma^{-}$ & -0.025 & 0.050 \\
\hline$\gamma p \rightarrow K^{0} \Sigma^{+}$ & -0.013 & 0.031 & $\gamma n \rightarrow K^{0} \Sigma^{0}$ & -0.019 & 0.031 \\
\hline Total & -0.063 & 0.105 & Total & 0.031 & 0.191 \\
\hline
\end{tabular}

\subsection{The anomalous magnetic moment of the nucleon}

One of the important ground state properties of the nucleon is the anomalous magnetic moment, which exists as a direct consequence of its internal structure. More than 30 years ago Gerasimov, and independently Drell and Hearn, proposed that this ground state property is related to the nucleon's resonance spectra by a sum rule which was then called the Gerasimov-Drell-Hearn (GDH) sum rule [6]. In the limit of photon point, the sum rule may be written as

$$
-\frac{\kappa_{N}^{2}}{4}=\frac{m_{N}^{2}}{8 \pi^{2} \alpha} \int_{0}^{\infty} \frac{d \nu}{\nu}\left[\sigma_{1 / 2}(\nu)-\sigma_{3 / 2}(\nu)\right]
$$

where $\sigma_{3 / 2}$ and $\sigma_{1 / 2}$ denote the cross sections for possible combinations of the nucleon and photon spins. Experiment with polarized beam and target has been performed at MAMI with photon energy up to $850 \mathrm{MeV}$ and data are being analyzed [7]. Using higher photon energies, experiments have been planned at ELSA and JLab.

For practical purpose, instead of Eq. (4) we use

$\kappa_{N}^{2}=\frac{m_{N}^{2}}{\pi^{2} \alpha} \int_{\nu_{\mathrm{thr}}}^{\nu_{\max }} \frac{d \nu}{\nu} \sigma_{T T^{\prime}}$

where $\sigma_{T T^{\prime}}$ denotes the cross section with polarized real photon and target. In terms of polarization observables this cross section corresponds to the double polarization $E$ [8]. Since there are no data available for $\sigma_{T T^{\prime}}$, previous work [9] approximated Eq. (5) with

$\kappa_{N}^{2} \leq \frac{m_{N}^{2}}{\pi^{2} \alpha} \int_{\nu_{\mathrm{thr}}}^{\nu_{\max }} \frac{d \nu}{\nu} \sigma_{T}$

to estimate the upper bound of contributions, where $\sigma_{T}$ represents the total cross section.

To calculate Eqs. (5) and (6) we use our elementary operator with $\nu_{\max }=2.2 \mathrm{GeV}$. The result is shown in Table 1. Our calculation yields values of $\kappa_{p}^{2}(K)=-0.063$ and $\kappa_{n}^{2}(K)=0.031$, or $\left|\kappa_{p}(K)\right| / \kappa_{p} \leq 0.14$ and $\kappa_{n}(K) / \kappa_{n} \leq 0.094$. This shows that the kaonhyperon final states contributions to the proton's and neutron's magnetic moment are very small. An interesting feature is that our calculation yields a negative contribution for the $\kappa_{p}^{2}(K)$ and a positive contribution for the $\kappa_{n}^{2}(K)$, which is obviously consistent with the result of Karliner's work [10]. 


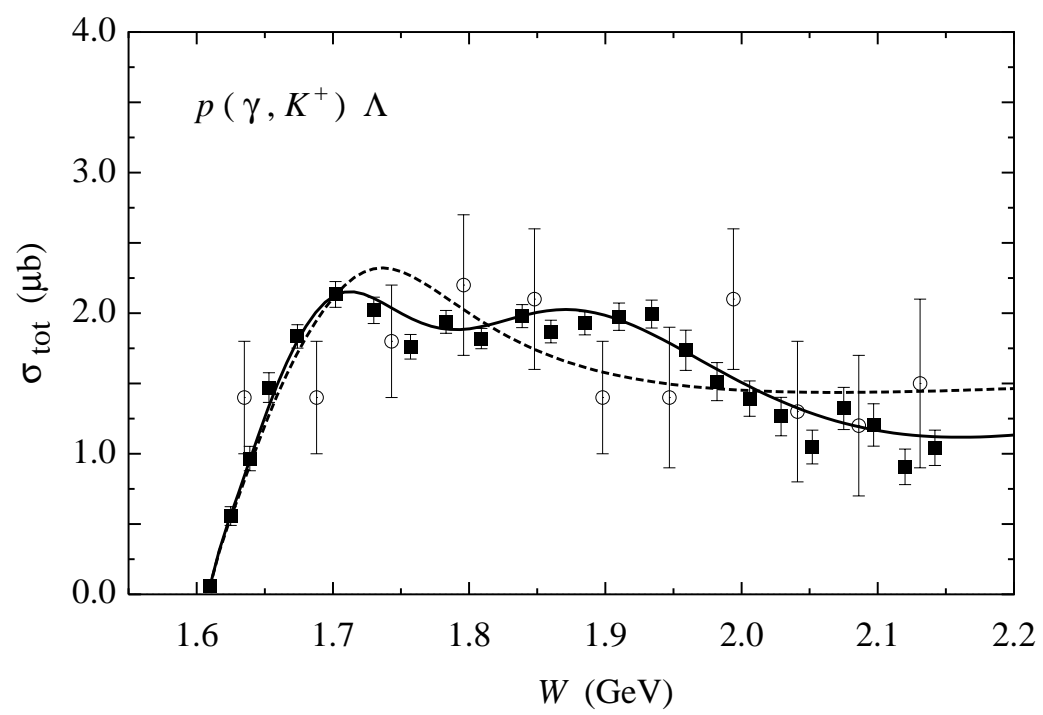

Figure 1. Total cross section for $K^{+} \Lambda$ photoproduction on the proton. The dashed line shows the model without the $D_{13}(1960)$ resonance, while the solid line is obtained by including the $D_{13}(1960)$ state. The new SAPHIR data [12] are denoted by the solid squares, old data are shown by the open circles.

\subsection{Investigation of missing resonances}

A brief inspection to the particle data book reveals that less than $40 \%$ of the predicted nucleon resonances are observed in $\pi N \rightarrow \pi N$ scattering experiments. Quark model studies have suggested that those "missing" resonances may couple strongly to other channels, such as the $K \Lambda$ and $K \Sigma$ channels [11]. Interestingly, the new SAPHIR total cross section data [12 for the $p\left(\gamma, K^{+}\right) \Lambda$ channel, shown in Fig. 1, indicate for the first time a structure around $W=1900 \mathrm{MeV}$. Using the current isobar model we investigate this structure. As shown in Fig. 1, our previous model cannot reproduce the total cross section. Although a structure in total cross section data does not immediately imply a new resonance, the energy region around $1900 \mathrm{MeV}$ represents a challenge not only because of possible broad, overlapping resonances, but also because there are additional production thresholds nearby, such as photoproduction of $\eta^{\prime}, K^{*} \Lambda$, and $K \Lambda^{*}$ final states, which can all lead to structure in the $K^{+} \Lambda$ cross section through final-state interaction. Here, we limit ourselves only to the possibility that this structure is in fact due to one of the missing or poorly known resonances.

The constituent quark model of Capstick and Roberts [11] predicts many new states around $1900 \mathrm{MeV}$. However, only a few of them have been calculated to have a significant $K \Lambda$ decay width [11. These are the $S_{11}(1945), P_{11}(1975), P_{13}(1950)$, and $D_{13}(1960)$ states. We have performed fits for each of these possible states, allowing the fit to determine the mass, width and coupling constants of the resonance. We found that all four states can reproduce the structure at $W$ around $1900 \mathrm{MeV}$, while reducing the $\chi^{2}$, but only for the $D_{13}(1960)$ state we found a remarkable agreement, up to the sign, between the quark model prediction and our extracted result [13]. The result is shown in Fig. 1, where without this resonance the model shows only one peak near threshold, while inclusion of the new resonance leads to a second peak at $W$ slightly below $1900 \mathrm{MeV}$, in accordance with the new SAPHIR data. The difference between the two calculations is much smaller for the differential cross sections. The largest effects are found in the photon asymmetry. Therefore, we would suggest that measuring this observable is well suited to shed more light on the contribution of this state in kaon photoproduction. 


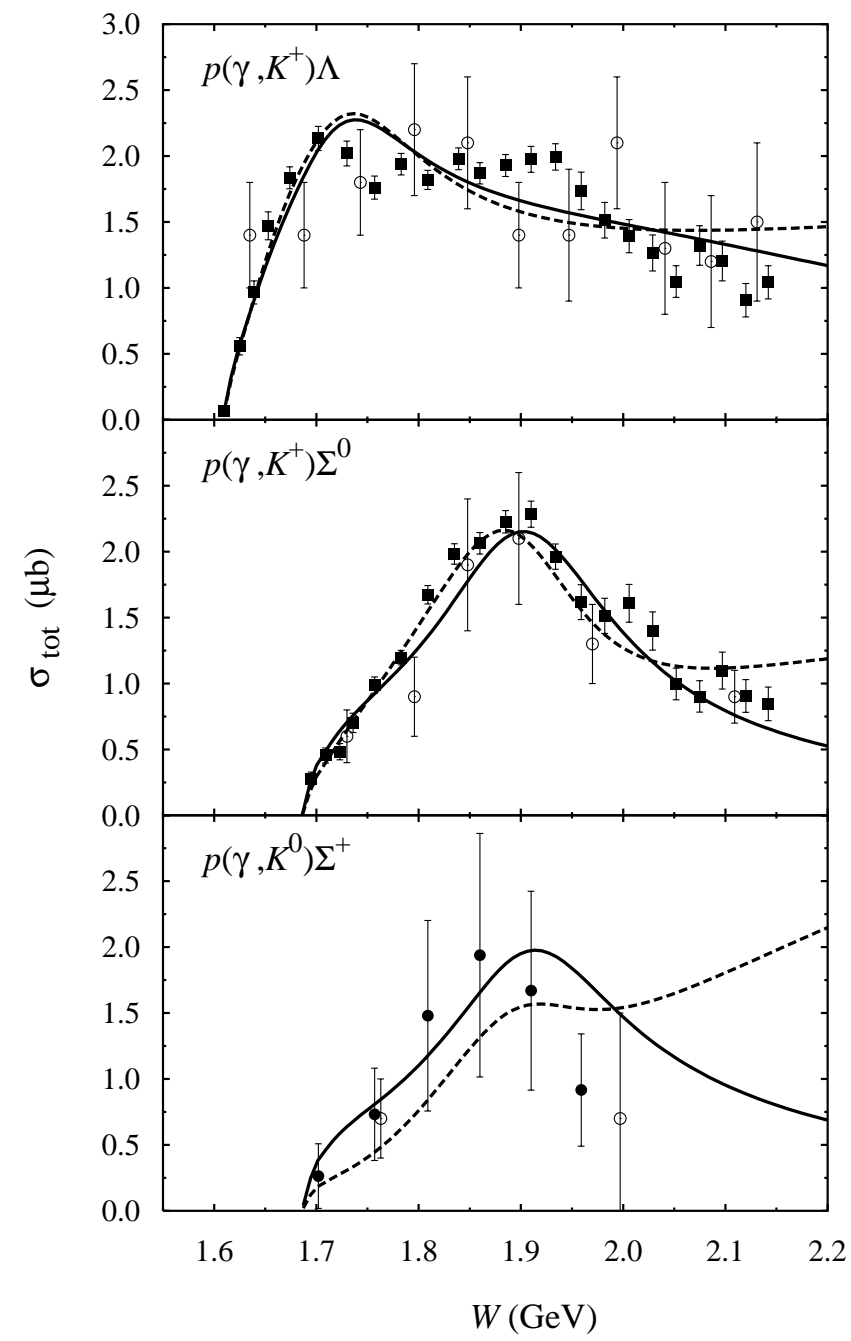

Figure 2. Total cross section for kaon photoproduction on the proton. The dashed line shows the isobar model with hadronic form factors, but without reggeization. The solid line is obtained by reggeizing the $K$ and $K^{*}$ propagators in the model. For $K^{+}$photoproduction, notation for the data is as in Fig. 1, for $K^{0}$ production old (open circles) and preliminary data (solid circles) are shown [14.

\section{EXTENSION TO HIGHER ENERGIES}

Extending the model to the higher energy regime requires a non-trivial task, since the Born terms increase rapidly as a function of energy. As shown in Fig. 2, even the hadronic form factors are unable to suppress the cross sections for the energy region above $2 \mathrm{GeV}$ as demanded by the data. Especially in the case of $K^{0} \Sigma^{+}$production, where the predicted cross section starts to monotonically increase at this point. However, in order to explore the higher-lying nucleon resonances or to account for higher energies contributions to the GDH integral, an isobar model which also properly work at higher photon energies would be demanded.

In Ref. 114 it has been shown that the contributions from the $t$-channel resonances are responsible for the divergence of the cross section, thus indicating that the Regge propagator should be used instead of the usual Feynman propagator. While a proper reggeization of the model is considerably complicated and the study is still underway, we investigate here only the qualitative effects of using Regge propagators in the model.

Following Ref. [15], we multiply the Feynman propagators $1 /\left(t-m_{K^{*}}^{2}\right)$ of the $K^{*}(892)$ 
and $K_{1}(1270)$ resonances in the operator with a factor of $P_{\text {Regge }} \cdot\left(t-m_{K^{*}}^{2}\right)$, where $P_{\text {Regge }}$ indicates the Regge propagator given in Ref. [15]. For the $K^{*}$ intermediate state it has the form

$P_{\text {Regge }}=\frac{s^{\alpha_{K^{*}}(t)-1}}{\sin \left[\pi \alpha_{K^{*}}(t)\right]} e^{-i \pi \alpha_{K^{*}}(t)} \frac{\pi \alpha_{K^{*}}^{\prime}}{\Gamma\left[\pi \alpha_{K^{*}}(t)\right]}$,

where $\alpha(t)=\alpha_{0}+\alpha^{\prime} t$ denotes the corresponding trajectory. Equation (7) clearly reduces to the Feynman propagator in the limit of $t \rightarrow m_{K^{*}}^{2}$, thus approximating the low energy behavior of the amplitude.

The model is then refitted to kaon photoproduction data and the result is shown in Fig. 2, where we compare the isobar model with and without reggeization. Obviously, Regge propagators strongly suppress the cross section at high energies and, therefore, yield a better explanation of data at this energy regime. For the $K^{0} \Sigma^{+}$process, the use of Regge propagators seems to give more flexibility in reproducing the cross section data. This cannot be achieved without reggeization, since the high energy behavior of both $t$-channel resonances is less controllable by the hadronic form factors. However, since the data for the $K^{0} \Sigma^{+}$channel shown in Fig. 22 are still preliminary [16], we have to wait before any further conclusion can be drawn. In future we will include the high energy data in the fit and investigate the model in the transition between medium and high energy regions.

\section{REFERENCES}

1. T. Mart, C. Bennhold, and C.E. Hyde-Wright, Phys. Rev. C 51 (1995) R1074.

2. C. Bennhold, T. Mart, and D. Kusno in Proceedings of the CEBAF/INT Workshop on $N^{*}$ Physics, Seattle, USA, 1996 (World Scientific, Singapore, 1997), p.166.

3. K. Ohta, Phys. Rev. C 40 (1989) 1335.

4. H. Haberzettl, C. Bennhold, T. Mart, and T. Feuster, Phys. Rev. C 58 (1998) R40.

5. H. Haberzettl, Phys. Rev. C 56 (1997) 2041.

6. S.B. Gerasimov, Sov. J. Nucl. Phys. 2 (1966) 430;

S.D. Drell and A.C. Hearn, Phys. Rev. Lett. 16 (1966) 908.

7. See W. Meyer, these proceedings.

8. R.L. Workman and R.A. Arndt, Phys. Rev. D 45 (1992) 1789.

9. D. Drechsel, Prog. Part. Nucl. Phys. 34 (1995) 181;

H.-W. Hammer, D. Drechsel, and T. Mart, nucl-th/9701008;

S. Sumowidagdo and T. Mart, Phys. Rev. C 60 (1999) 028201.

10. I. Karliner, Phys. Rev. D 7 (1973) 2717.

11. S. Capstick and W. Roberts, Phys. Rev. D 58 (1998) 074011.

12. SAPHIR Collaboration: M.Q. Tran et al., Phys. Lett. B 445 (1998) 20.

13. T. Mart and C. Bennhold, Phys. Rev. C 61 (2000) 012201(R).

14. F.X. Lee, T. Mart, C. Bennhold, H. Haberzettl, and L.E. Wright, nucl-th/9907119 and references therein.

15. M. Guidal, J.M. Laget, and M. Vanderhaeghen, Nucl. Phys. A 627 (1997) 645.

16. By the time of finishing this paper we realized that the final version of SAPHIR data for the $\gamma+p \rightarrow K^{0} \Sigma^{+}$channel has been published in: S. Goers et al., Phys. Lett. B 464 (1999) 331. 